\title{
Lattice Expansion of (Ga,Mn)As: The Role of Substitutional Mn and of the Compensating Defects
}

\author{
J. MAŠEK AND F. MÁCA \\ Institute of Physics, AS CR, Na Slovance 2, 18221 Prague 8, Czech Republic
}

\begin{abstract}
We apply the density-functional technique to determine the lattice constant of GaAs supercells containing $\mathrm{Mn}_{\mathrm{Ga}}, \mathrm{Mn}_{\mathrm{int}}$, and $\mathrm{As}_{\mathrm{Ga}}$ impurities, and use a linear interpolation to describe the dependence of the lattice constant $a$ of $\mathrm{Ga}_{1-x} \mathrm{Mn}_{x}$ As on the concentrations of these impurities. The results of the supercell calculations confirm that $\mathrm{Mn}_{\mathrm{Ga}}$ does not contribute to the lattice expansion. The increase in $a$ is due to both $\mathrm{Mn}_{\text {int }}$ and $\mathrm{As}_{\mathrm{Ga}}$, that are both created in the as-grown (Ga,Mn)As in proportion to $x$, and that are most probably present in a remarkable amount also in the best annealed materials.
\end{abstract}

PACS numbers: 71.15.Ap, 71.20.Nr, 71.55.Eq, 75.50.Pp

\section{Introduction}

Diluted magnetic semiconductors (DMS), represented by (Ga,Mn)As mixed crystals, are particularly interesting materials because of their hole-mediated ferromagnetism $[1,2]$. This, combined with the semiconducting behavior, makes the DMS attractive for applications in spin electronics, and stimulated extensive theoretical and experimental studies of these materials in the last years.

Recently, the compositional dependence of the lattice constant of the (Ga,Mn)As mixed crystals has been investigated with respect to Mn incorporation in the lattice. It has been known for long that the lattice constant of $(\mathrm{Ga}, \mathrm{Mn}) \mathrm{As}$ increases with increasing content of Mn [3]. Originally, it was attributed to the substitution of Mn for Ga in the cation sublattice and it was assumed that the lattice constant of (Ga,Mn)As extrapolates to the lattice constant of the zinc-blende MnAs crystal that is larger than the lattice constant of GaAs [4]. This assumption is, however, in a contradiction with the simple estimate based on the atomic radii of the constituent atoms because $\mathrm{Mn}$ atom is smaller $(0.117 \mathrm{~nm})$ than Ga atom $(0.125 \mathrm{~nm})[5]$. Also the density-functional study of $\alpha$-MnAs showed [6] that the 
lattice constant of MnAs with a perfect zinc-blende structure is smaller than the lattice constant of GaAs.

Most recently, the density-functional method of determining the lattice constant by minimizing the total energy has been applied also to the (Ga,Mn)As. The mixed crystals with realistic concentrations of $\mathrm{Mn}$ up to 10 percent were treated by means of the linear muffin-tin orbital (LMTO) method within the coherent-potential approximation (CPA) [7]. It was found that the substitutional $\mathrm{Mn}\left(\mathrm{Mn}_{\mathrm{Ga}}\right)$ has only a negligible effect on the lattice constant in this concentration range. It was deduced that the observed increase in the lattice parameter is a secondary effect caused by creation of an increasing number of compensating donors, proportionally to the content of Mn. In particular, the As antisite defects $\left(\mathrm{As}_{\mathrm{Ga}}\right)$ and $\mathrm{Mn}$ atoms in the interstitial positions $\left(\mathrm{Mn}_{\mathrm{int}}\right)$ were shown to cause a remarkable increase in the lattice constant of (Ga,Mn)As. Quantitatively, the dependence of the lattice parameter $a\left(x_{s}, x_{i}, y\right)$ on the partial concentrations of $\mathrm{Mn}_{\mathrm{Ga}}, \mathrm{Mn}_{\mathrm{int}}$, and $\mathrm{As}_{\mathrm{Ga}}$ was parametrized in the following form:

$$
a\left(x_{s}, x_{i}, y\right)=a_{0}+0.002 x_{s}+0.105 x_{i}+0.069 y(\mathrm{~nm}),
$$

where $a_{0}$ is the lattice constant of the pure GaAs.

In strongly compensated materials it is expected that the number of $\mathrm{Mn}_{\text {int }}$ and $\mathrm{As}_{\mathrm{Ga}}$ increases proportionally to the total concentration $x=x_{s}+x_{i}$ of $\mathrm{Mn}$ [8]. The dependence of $a$ on $x$ can be simply estimated from Eq. (1) in the limiting case of the complete compensation, i.e. for $x_{s}=2 x_{i}+2 y$. In this case, we obtain a linear dependence $a(x) \approx a_{0}+0.035 x(\mathrm{~nm})$. The coefficient will be smaller for partial compensation which is in reasonable agreement with the experimental value of $0.032 \mathrm{~nm}[3]$.

The theoretical prediction that the increase in the lattice parameter $a$ with Mn concentration is, at least partly, connected with the presence of the interstitial Mn, was also confirmed by recent measurements [9-11] in which the lattice constants for as-grown and annealed materials were compared. During the post-growth thermal treatment, the interstitial Mn atoms diffuse out of the material and the lattice constant decreases accordingly. These measurements, however, differ in several respects from the calculations. First of all, the calculated increase in the lattice constant due to $\mathrm{Mn}_{\text {int }}$ according to Eq. (1) seems overestimated by a factor of 2 . Second, even the best materials with minimum compensation (i.e. with all interstitials removed) have their lattice constant larger than GaAs and increasing with the Mn content. It is interpreted that - after all - the Mn substitution itself can expand the lattice.

That is why we re-examine our original coherent-potential study [7] by using the full-potential linearized-augmented-plane-wave method (FPLAPW [12]) that overcomes some simplifications involved in the LMTO-CPA study (atomic-sphere approximation, unrelaxed lattice, etc.). We apply it to the supercells of GaAs with $\mathrm{Mn}_{\mathrm{Ga}}, \mathrm{Mn}_{\mathrm{int}}$, and $\mathrm{As}_{\mathrm{Ga}}$ impurities. 


\section{Results}

We use tetragonal supercells formed by 8 and 16 molecular units of GaAs. These supercells, containing a single defect $\left(\mathrm{Mn}_{\mathrm{Ga}}, \mathrm{Mn}_{\mathrm{int}}\right.$, or $\left.\mathrm{As}_{\mathrm{Ga}}\right)$, represent materials with 12.5 and 6.25 atomic percent of the impurities, respectively. The lattice constant was determined by minimizing the total energy $E_{\text {tot }}$ of the supercell with respect to $a$, either with or without lattice relaxation. In practice, the dependence of $E_{t o t}$ on $a$ was approximated by a cubic polynomial fitted in approx. 10 points.

The results are summarized in Fig. 1. First of all, the calculations confirm that the substitutional $\mathrm{Mn}_{\mathrm{Ga}}$ atoms do not contribute to the expansion of the lattice. The dependence of the lattice constant on $x_{s}$ is weak and, in contrast to the CPA results, decreasing. Assuming that the lattice constant of ( $\mathrm{Ga}, \mathrm{Mn})$ As follows the Vegard law, i.e. that the function $a\left(x_{s}\right)$ is linear in the entire concentration range, we obtain the linear coefficient $\approx-0.005 \mathrm{~nm}$. This value is in reasonable agreement with the interpolation between the lattice constants obtained for GaAs and $\alpha$-MnAs [6].

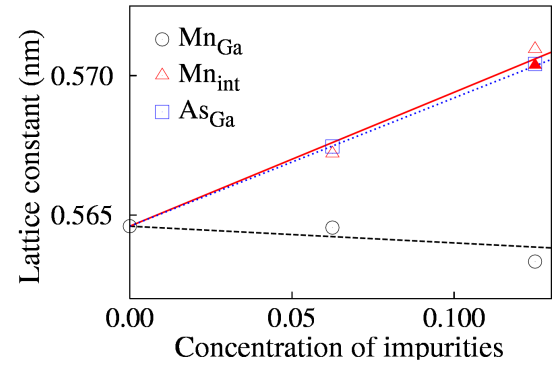

Fig. 1. Calculated lattice constant as a function of the concentration of the impurities: Mn atoms in the substitutional positions (circles), Mn atoms in the interstitial positions (triangles), As antisite defects (boxes). The full triangle corresponds to a relaxed geometry.

The interstitial Mn atoms were considered in both tetrahedral positions, $\mathrm{T}\left(\mathrm{As}_{4}\right)$ and $\mathrm{T}\left(\mathrm{Ga}_{4}\right)$. Although the local relaxation of the lattice is different for these two defects [13], the effect on the lattice expansion is almost the same for both. That is why only the data for $\mathrm{T}\left(\mathrm{As}_{4}\right)$ are presented in Fig. 1. Without relaxation (empty triangles), the lattice expands significantly in the presence of $\mathrm{Mn}_{\text {int }}$ and the lattice constant increases almost linearly with $x_{i}$. If the relaxation around $\mathrm{Mn}_{\text {int }}$ is taken into account, the expansion is slightly weaker, as indicated by a full triangle in Fig. 1. It is important to notice that the linear coefficient is $\approx 0.048 \mathrm{~nm}$ now, approximately one half of the value obtained from the CPA calculations.

The contribution of the $\mathrm{As}_{\mathrm{Ga}}$ antisite defects to the lattice expansion is very similar to $\mathrm{Mn}_{\mathrm{int}}$. Assuming that the contributions of various defects to the lattice 
expansion are additive, we summarize the above results into a simple linear formula

$$
a\left(x_{s}, x_{i}, y\right)=a_{0}-0.005 x_{s}+0.048 x_{i}+0.046 y(\mathrm{~nm}) .
$$

\section{Discussion}

Equation (2) is a full-potential counterpart of Eq. (1) that was obtained from the TB-LMTO-CPA study [7]. Because of several simplifications involved in the CPA calculations (effective medium, minimum basis, atomic-sphere approximation, etc.) Eq. (2) should be considered more reliable. At the same time, however, the sensitivity of the parameters of Eqs. $(1,2)$ to the method of calculations indicates that also the coefficients in Eq. (2) represent only a rough quantitative estimate for the compositional dependence of the lattice constant and should be used with a caution.

The most important features of Eq. (2) is that it confirms the recent results $[6,7]$ that the lattice expansion of $(\mathrm{Ga}, \mathrm{Mn})$ As cannot be attributed to the substitutional $\mathrm{Mn}$. It is related to the presence of the interstitial $\mathrm{Mn}$ and $\mathrm{As}_{\mathrm{Ga}}$ antisite defects as well. The contribution of these two intrinsic defects to the expansion is characterized by a linear coefficient $\approx 0.05 \mathrm{~nm}$ instead of much larger values resulting from the CPA calculations. The present results are much closer to the experiment [9-11].

To obtain a more detailed picture of the lattice expansion of real materials and to compare the role of $\mathrm{Mn}_{\text {int }}$ and $\mathrm{As}_{\mathrm{Ga}}$ we need to estimate the partial concentrations of $\mathrm{Mn}_{\mathrm{Ga}}, \mathrm{Mn}_{\mathrm{int}}$, and $\mathrm{As}_{\mathrm{Ga}}$. To do this, we start with the concentration dependent formation energies $E\left(\mathrm{Mn}_{\mathrm{Ga}}\right), E\left(\mathrm{Mn}_{\mathrm{int}}\right)$, and $E\left(\mathrm{As}_{\mathrm{Ga}}\right)[8,14]$ and assume that the corresponding partial concentrations in the as-grown (Ga,Mn)As can be approximately calculated from the dynamical-equilibrium conditions $[8,15]$

$$
E\left(\mathrm{Mn}_{\mathrm{Ga}}\right)=E\left(\mathrm{Mn}_{\mathrm{int}}\right), \quad E\left(\mathrm{As}_{\mathrm{Ga}}\right)=0 .
$$

Using for simplicity the linearized form of the formation energies we obtain that, except for the lowest values of $x$, the partial concentrations of substitutional and interstitial $\mathrm{Mn}$, and also the concentration of the $\mathrm{As}_{\mathrm{Ga}}$ antisite defects increase proportionally to $x$,

$$
x_{s} \approx 0.85 x, \quad x_{i} \approx 0.15 x, \quad y \approx 0.11 x .
$$

Combining Eq. (2) with the estimate Eq. (4), we arrive to the theoretical model of the concentration dependence of the lattice constant in the as-grown $(\mathrm{Ga}, \mathrm{Mn})$ As. It is shown by the solid line in Fig. 2. The dotted and dashed lines in Fig. 2 correspond to the annealed materials with a reduced amount of the interstitial $\mathrm{Mn}$, but with the unchanged concentration of the more stable $\mathrm{Mn}_{\mathrm{Ga}}$ and $\mathrm{As}_{\mathrm{Ga}}$. If all $\mathrm{Mn}_{\text {int }}$ are removed, the lattice constant is still an increasing function of $x$, but its slope is much smaller. An intermediate case with the number of the Mn interstitials reduced to one third is considered as a realistic example. Most probably, the observed increase in the lattice constant in the annealed mater- 


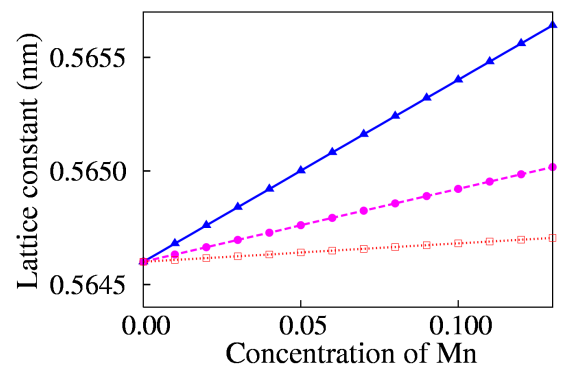

Fig. 2. Concentration dependent lattice constant of (Ga,Mn)As according to Eqs. (2) and (4); as-grown material with $\mathrm{Mn}_{\mathrm{Ga}}, \mathrm{Mn}_{\mathrm{int}}$, and $\mathrm{As}_{\mathrm{Ga}}$ (solid line), mixed crystal with all $\mathrm{Mn}_{\text {int }}$ removed (dotted), material with the $x_{i}$ reduced to one third (dashed).

ials [9-11] indicates that a large number of the compensating antisite defects $\left(\mathrm{As}_{\mathrm{Ga}}\right.$ as well as some residual $\mathrm{Mn}_{\text {int }}$ ) are present even in the best annealed samples with the minimum compensation.

To summarize, the full-potential supercell calculations confirmed qualitatively the recent results of the CPA studies [7] of the concentration dependence of (Ga,Mn)As mixed crystals. The present results are closer to the experiment. They show the negligible influence of the substitutional Mn on the lattice constant and the role of the intrinsic compensating donors in the lattice expansion.

\section{Acknowledgment}

This work has been done within the project AVOZ1-010-0520 of the AS CR. The financial support was provided by the Academy of Sciences of the Czech Republic (Grant No. A1010214) and by the Grant Agency of the Czech Republic $(202 / 04 / 583)$.

\section{References}

[1] H. Ohno, Science 281, 951 (1998).

[2] T. Dietl, H. Ohno, F. Matsukura, J. Cibert, D. Ferrand, Science 287, 1019 (2000).

[3] H. Ohno, J. Magn. Magn. Mater. 200, 110 (1999).

[4] G.M. Schott, G. Schmidt, G. Karczewski, L.W. Molenkamp, R. Jakiela, A. Barcz, G. Karczewski, Appl. Phys. Lett. 82, 4678 (2003).

[5] J.A. Dean, Langes' Handbook of Chemistry, Tab.3-118, McGraw-Hill, New York 1973.

[6] Yu-Jun Zhao, W.T. Geng, A.J. Freeman, B. Delley, Phys. Rev. B 65, 113202 (2002).

[7] J. Mašek, J. Kudrnovský, F. Máca, Phys. Rev. B 67, 153203 (2003).

[8] J. Mašek, I. Turek, J. Kudrnovský, F. Máca, V. Drchal, Acta Phys. Pol. A 105, 637 (2004). 
[9] I. Kuryliszyn-Kudelska, J.Z. Domagala, T. Wojtowicz, X. Liu, E. Lusakowska, W. Dobrowolski, J.K. Furdyna, J. Appl. Phys. 95, 603 (2004).

[10] J. Sadowski, J.Z. Domagala, Phys. Rev. B 69, 075206 (2004).

[11] L.X. Zhao, C.R. Staddon, K.Y. Wang, K.W. Edmonds, R.P. Campion, B.L. Gallagher, C.T. Foxon, Appl. Phys. Lett. 86, 071912 (2005).

[12] P. Blaha, K. Schwarz, J. Luitz, WIEN97, FPLAPW package for calculating crystal properties, TU Vienna.

[13] J. Mašek, F. Máca, Phys. Rev. B 69, 165212 (2004).

[14] J. Mašek, I. Turek, V. Drchal, J. Kudrnovský, F. Máca, Acta Phys. Pol. A 102, 673 (2002).

[15] J. Mašek, F. Máca, to be published. 